\title{
Stufen zu einer religiösen Metaphorik. Der ,andere' Cohen in Skizzen eines Editors
}

\author{
Wiedebach, Hartwig
}

\begin{abstract}
Hermann Cohen s passionate philosophizing begins with a departure from the letter of the rabbinical doctrine of revelation. Initially his Science of Reason is shaped by a psychology of language based on Plato, Herder, Humboldt, and Steinthal. Later the influence of Kant is prevalent. In the end Cohen s System of Philosophy becomes the foundation upon which he reappropriates the sources of Judaism in their literalness. His program from 1908/09 onwards is to think the uniqueness of God as it must be felt. A distinctive metaphorics comes into being: a hermeneutics of being human anchored in Reason through comparison with an incomparable God. The critical edition of his works illuminates this philosophical process by means of details previously not taken into account
\end{abstract}

DOI: https://doi.org/10.1524/dzph.2011.0022

Posted at the Zurich Open Repository and Archive, University of Zurich ZORA URL: https://doi.org/10.5167/uzh-154943

Journal Article

Published Version

Originally published at:

Wiedebach, Hartwig (2011). Stufen zu einer religiösen Metaphorik. Der ,andere‘ Cohen in Skizzen eines Editors. Deutsche Zeitschrift für Philosophie, 59(2):295-309.

DOI: https://doi.org/10.1524/dzph.2011.0022 


\title{
Stufen zu einer religiösen Metaphorik
}

\author{
Der , andere' Cohen in Skizzen eines Editors
}

Von HARTWIG WIEDEBACH (Zürich)

In Erinnerung an Dieter Adelmann

(1936-2008)

Hermann Cohen war ein deutsch-jüdischer Denker und Stilist aus Leidenschaft. Man nennt ihn einen „Neukantianer“. Das schadet indes nicht weniger, als es nützt, denn dieses Etikett steht meist für eine einseitige Fokussierung auf Geltungs- beziehungsweise Wissenschaftslogik. „Neukantianismus“ ist mithin ein Klassifikationsbegriff, den man je nach Einstellung affirmativ oder pejorativ besetzt. Davon nehme ich Abstand. Nicht dass ich die wissenschaftslogische Seite herabstufen wollte. Aber in und neben Cohens Wissenschaft mischen sich Momente des Erzählens, des Bekennens, der Rhetorik ein, die von seinem philosophischen Profil untrennbar sind. Wer würde seine lebenslange Faszination für Ideenschau und Prophetie „Neukantianismus“ nennen? Oder seine Lehre vom „reinen Gefühl“ mit ihren Motiven in der Sprachpsychologie Herders, Humboldts, Steinthals und Moritz Lazarus'? Oder seine Besinnung auf die Quellen des Judentums, die in der Versöhnung (kippur) des ,absoluten Individuums“ mit Gott und einer liturgisch gestalteten „Korrelation“ kulminiert? Zweifellos: Cohen hat sich mit Nachdruck als Kantianer bekannt, und auch die Schule in Marburg war ihm wichtig. Die Quellen seines „Marburg“ aber sowie das, was dort und später in Berlin zum Ausdruck kam, waren mehr als Neukantianismus. Sein Schreiben ist ein unablässiges Philosophieren in statu nascendi: eine Dauerspannung zwischen System und gelebtem Leben. Die Art, wie Cohen diesen Weg geht, macht ihn zeitlos. Ich nutze neuere Ergebnisse der kritischen Werkedition für eine Skizze, die mit keiner Klassifikation etwas zu tun hat. ${ }^{1}$

1 Cohens Werke werden seit 1977 von Helmut Holzhey, inzwischen unter Beteiligung des Verfassers, bei Olms in Hildesheim herausgegeben: 11 Bände Monographien (Bde. 1-10 sind erschienen, es fehlt noch Bd. 11: Religion der Vernunft aus den Quellen des Judentums), 6 Bände Kleinere Schriften in chronologischer Folge, durchgehend kommentiert (Bde. 12-17, die letzten drei liegen vor), 4 Bände Supplementa (Bd. 1, Reflexionen und Notizen, und Bd. 2, Die Hermann-Cohen-Bibliothek, sind erschienen). Briefbände folgen. 


\section{Judentum und Philosophie}

Mein Ausgangspunkt ist die auffälligste Spannung in Leben und Werk Cohens, nämlich die zwischen systematischer Philosophie und jüdischem Glauben. Hier dokumentiert die Werkausgabe eine Grundsatzentscheidung. Sie meidet die Trennung der Bereiche. Das ist nicht selbstverständlich: Immer noch werden Cohens Aufsätze überwiegend anhand zweier älterer Sammlungen unter programmatisch verschiedenen Titeln rezipiert, Jüdische Schriften und Schriften zur Philosophie und Zeitgeschichte. ${ }^{2}$ Zwar wurden beide von der 1919 gegründeten Berliner Akademie für die Wissenschaft des Judentums herausgegeben, aber die Einheit der Institution hat die nachhaltige Wirkung der Bereichstrennung nicht verhindert. Die Werkausgabe präsentiert die Kleineren Schriften stattdessen in chronologischer Folge, textkritisch und inhaltlich durchgehend kommentiert. Der Kalender ersetzt die Bereichslogik. Wir unterstellen die biographische Einheit.

Unter den zeitgenössischen Berichten über Cohen ragen zwei heraus: die Broschüre Hermann Cohen aus persönlicher Erinnerung von Robert Arnold Fritzsche und die Einleitung zu Cohens Jüdischen Schriften von Franz Rosenzweig. ${ }^{3}$ Fritzsche, klassischer Philologe und Bibliothekar in Gießen, ziseliert in feiner Präzision die intellektuelle Physiognomie Cohens, mit Betonung der Zwischentöne in einem vielseitigen Treiben und Getriebensein. Philosoph sein und Jude sein sind hier zwei Nuancen desselben Eros. Rosenzweig, Hegelforscher und jüdischer Sprachdenker, präsentiert Cohen als impulsiven Denker des 19. Jahrhunderts, der trotz Antisemitismus dem Deutschtum Kants und Goethes dient, der noch einmal ein System entwirft, und der doch zuletzt - beim Wechsel 1912 von Marburg an die Berliner Lehranstalt für die Wissenschaft des Judentums - zu ,seinen“ Juden zurückkehrt, deren Glauben er als junger Mann mit der Philosophie vertauscht hatte. Beide Autoren haben Recht: Es gibt sowohl die Kontinuität in kaum bestimmbaren Übergängen als auch die in Leidenschaft und Entscheidungen verdichtete Polarisierung.

Hermann Jecheskel Cohen wurde am 4. Juli 1842 in dem anhalt-bernburgischen Städtchen Coswig geboren und starb am 4. April 1918 in Berlin. Ich zeichne nur ein einzelnes Detail seiner Jugend. Die ersten Grundlagen jüdischer und allgemeiner Bildung erwarb er bei seinem Vater Gerson Cohen, Kantor und Lehrer in der Coswiger israelitischen Gemeinde. Im Oktober 1857 bezog Hermann das 1854 eröffnete, später berühmte Rabbinerseminar Fraenckelscher Stiftung in Breslau. Seine Lehrer waren bedeutende Vertreter der 30 Jahre zuvor begründeten „Wissenschaft des Judentums“: so der Talmudist Zacharias Frankel, der Historiker Heinrich Graetz, der klassische Philologe Jacob Bernays und der Philosophiehistoriker Manuel Joël. Es dauerte nicht lange, und Cohen stand vor einer Entscheidung. Zacharias Frankel hatte 1859 eine Einführung in die Mischna, den grundlegenden Kanon von Verordnungen des talmudischen Judentums, veröffentlicht. ${ }^{4}$ Seine Methode vereinte die historische Kritik mit dem Glauben an die göttliche Offenbarung. Das provozierte die damalige Neuorthodoxie um den Frankfurter Rabbiner Samson Raphael Hirsch. Anlass waren unter anderem religiöse Vorschriften, die

2 Vgl. H. Cohen, Jüdische Schriften, 3 Bde., Berlin 1924; ders., Schriften zur Philosophie und Zeitgeschichte, 2 Bde., Berlin 1928.

3 Vgl. R. A. Fritzsche, Hermann Cohen aus persönlicher Erinnerung, Berlin 1922; F. Rosenzweig, Einleitung, in: H. Cohen, Jüdische Schriften, a. a. O., Bd. 1, XIII-LXIV (+ Anmerkungen, 331335), auch in: F. Rosenzweig, Gesammelte Schriften, III: Zweistromland. Kleinere Schriften zu Glauben und Denken, Dordrecht 1984, 177-223.

4 Z. Frankel, Darke ha-Mischna. Hodegetica in Mischnam [...], pars prima: Introductio in Mischnam, Leipzig 1859. 
sich zwar erst im Talmud, also noch nicht in der hebräischen Bibel finden, die aber dennoch als halachot le-Mosche mi-Sinaï, als „Verordnungen an Mose vom Sinai“ gelten und deshalb dieselbe Offenbarungsautorität wie die Gebote der Tora haben. Frankel schloss nicht völlig aus, dass kontingente historische Umstände bei diesen Verordnungen eine Rolle gespielt haben mochten. Seine Gegner verglichen ihn daraufhin mit Götzendienern und Sabbatschändern. ${ }^{5}$

Cohen hält den Streit für sinnlos. Für ihn bedeutet das Gütesiegel ,,an Mose vom Sinai“ „nichts Anderes, als: die betreffenden talmudischen Verordnungen sind dem Geiste der mosaischen Gesetzgebung gemäß“; es ist keine Entweihung, wenn sie „,bei dieser und jener Gelegenheit durch diesen oder jenen Mann entstanden sind“. ${ }^{6}$ In dieser Klarheit sagt er das allerdings erst vier Jahre später; zunächst betraf ihn die Angelegenheit in Unsicherheit und „inneren Kämpfen“. Schließlich aber findet er zu seinem Selbstverständnis. Er begreift sich nun als Wissenschaftler dessen, was er soeben „Geist“" nannte. Die „mosaische Gesetzgebung“ ist eine von dessen Erscheinungen: Ihrem „Geist“ nach ist sie ein „weltgeschichtlicher Prozeß [...], in dem ein Glied ins andere eingefügt ist" “. ${ }^{7}$ Das buchstäbliche Fürwahrnehmen der Tradition ist also für Cohen verblasst, nicht jedoch seine Bindung an die jüdische Gemeinschaft. Gerade seine Auffassung von den historischen Realisierungen des Geistes hält ihn daran fest, denn diese Gemeinschaft spielt eine grundlegende kultur- und sprachformende Rolle. Sie ist und bleibt, wie er Jahre später bekennt, Bewahrerin eines „der Vermenschlichung unzugänglichen Kerns des alten Prophetengottes“ “. ${ }^{8}$ Das prägt Cohens Begriff des Geistes. Gerade als Jude lebt er unter der Aufgabe, die Unvergleichbarkeit und Einzigkeit Gottes als Motor dieses Geistes darzustellen. Das bedeutet für ihn, eine Metamorphose der göttlichen Offenbarung in Vernunfterkenntnis zu vollziehen. So gelangt Cohen zur Philosophie. „Die auswärtige Quelle fließt unversehens in eine eigene über: in die der menschlichen Vernunft, sofern der Begriff der Vernunft den Menschen mit Gott vereinbart und versöhnt", schreibt er 40 Jahre später in seiner Ethik des reinen Willens. ${ }^{9}$

Die letzte Konsequenz zieht ein Satz aus der nochmals 15 Jahre späteren Religion der Vernunft aus den Quellen des Judentums: „Die Offenbarung ist die Schöpfung der Vernunft."10 Hier, 60 Jahre nach seinem Eintritt in das Rabbinerseminar, kehrt Cohen zur theologischen Sprache zurück und entdeckt seinen eigenen Stil im philosophischen Kommentieren von Tora und Talmud, durch den er sich die alten Stoffe erneut in ihrer Buchstäblichkeit zueignet. „Vernunft", so heißt es nun, „erschöpft sich nicht in Wissenschaft und Philosophie." ${ }^{11}$ Sie tritt in religiösen Quellen hervor: den „Quellen des Judentums“. Deren Zentrum ist - ursprünglicher als in Christentum und Islam ${ }^{12}$ - die Einzigkeit Gottes. Sie sind das „Material [...], in dessen geschichtlicher Selbsterzeugung die problematische Vernunft, die Religion der Vernunft sich

5 Vgl. dazu: Jeschurun, 7 (1861), Januarheft, 201 u. 211, zit. in: H. Cohen, Kleinere Schriften, I, Werke, 12, Einleitung, $X$.

6 Brief an Eduard Steinthal ca. 1865, ein Rückblick vier Jahre nach dem Streit, in: H. Cohen, Briefe, hg. v. Bertha u. Bruno Strauß, Berlin 1939, 13 u. 14.

7 Ebd., 14.

8 H. Cohen, Ein Bekenntnis in der Judenfrage [1880], Kleinere Schriften, II, vorerst noch: Jüdische Schriften, II, 77.

9 Ders., Ethik des reinen Willens [1904, 1907²], Werke, 7, 54.

10 Ders., Religion der Vernunft aus den Quellen des Judentums (Nachdruck der 2. Aufl., 1929), Wiesbaden $1978,84$.

11 Ebd., 8.

12 Vgl. (unter anderem zu Lessings Ringparabel) H. Cohen, Reflexionen und Notizen, Werke, Suppl. 1, Blätter 1 u. 70; sowie ders., Religion der Vernunft, a. a. O., 39. 
erzeugen und bewahrheiten soll““. ${ }^{13}$ Quellen sind mithin Vollzug einer Autopoiesis. Deren Organ ist die religiöse „Nationalität" ${ }^{\text {"14 }}$, traditionell gesprochen das jüdische Volk. Die sich in den Quellen und ihren Kommentaren vollziehende „Schöpfung der Vernunft“ strebt aus sich heraus über jede dogmatisch-kasuistische Engführung hinaus.

Am Anfang dieses Weges aber stand Cohens Abschied vom Seminar, der, wie ihm immer bewusst blieb, ,,vornehmsten Bildungsstätte seiner Jugend“. ${ }^{15}$ Nach der philosophischen Initialzündung noch Rabbiner werden zu wollen, erschien ihm als „Heuchelei“. ${ }^{16}$ Mit einer soliden klassischen Sprachbildung durch Jacob Bernays und „einem ehrenvollen Zeugniß, das von Frankel, Bernays, Joel, Zuckermann und Graetz unterzeichnet war ${ }^{617}$, bezog er nacheinander die Universitäten Breslau (Herbst 1861) und Berlin (Herbst 1864). Im September 1865 wurde er extern in Halle im Fach „Klassische Philosophie \& Geschichte“ promoviert. ${ }^{18}$ Durch umfangreiche Artikel in Moritz Lazarus' und Chajim H. Steinthals Zeitschrift für Völkerpsychologie und Sprachwissenschaft begann er sich einen Namen zu machen.

\section{Psychologie der inneren Sprachform}

„Aller Fortschritt der Philosophie beruht auf der Psychologie“ (omnem philosophiae progressum in psychologia constitutum esse) war die Hauptthese der Dissertation Philosophorum de antinomia necessitatis et contingentiae doctrinae (1865). ${ }^{19}$ Sie blieb im Großen und Ganzen bis zu Cohens erstem Kantbuch Kants Theorie der Erfahrung (1871) gültig. Die Psychologie nahm in diesen Jahren immer deutlicher den Charakter einer Sprachpragmatik im Sinn von Chajim Steinthal an. Spätestens mit Kants Begründung der Ethik von 1877 trat sie in den Hintergrund. Jahrzehnte später aber kehrte sie in Cohens Theoriebildung zurück - nun mit einem schärfer gefassten Begriff der Sprache und ohne den Anspruch, allen Fortschritt der Philosophie darauf zu gründen.

Man kann in Band I der Kleineren Schriften, dessen Kommentar die Texte bis 1869 auf ihre Quellen hin durchsichtig macht, deutlicher als bisher das Hervortreten dieser Sprachwissenschaft erkennen. ${ }^{20}$ Indikatoren sind die Rezeption von Herders Gleichnis-Psychologie und die immer präzisere Frage nach der metaphorischen Dynamik in Platons „Idee“. Schon die Dissertation spricht beides an, aber eher verhalten und ohne Zusammenhang. ${ }^{21}$ In der

13 Ders., Religion der Vernunft, a. a. O., 5.

14 Ebd., 28, 422.

15 H. Cohen, Ein Gruß der Pietät an das Breslauer Seminar [1904], Kleinere Schriften, III; vorerst noch: Jüdische Schriften, II, 418.

16 So die Erinnerung eines Mitseminaristen: A. Moses, Prof. Dr. Hermann Cohen in Marburg und sein Bekenntniß in der Judenfrage. Eine Reminiszenz und Kritik, Milwaukee/Wisconsin 1880, 4; demnächst in: H. Cohen, Kleinere Schriften, II, Werke, 13.

17 Ebd., 4.

18 Vgl. H. Cohen, Kleinere Schriften, I, Werke, 12 (im Druck), Einleitung, XV f.

19 Ders., Kleinere Schriften, I, 1-39 (lat.), zit. 39. - Der Band enthält eine deutsche Übersetzung der Diss. durch die Herausgeber, 41-91, zit. 91.

20 Ders., Werke, 12. Zu ergänzen sind die Befunde aus Cohens Privatbibliothek; vgl. H. Wiedebach, Die Hermann-Cohen-Bibliothek (= Cohen, Werke, Suppl. 2). - Der Band führt nur Schriften auf, die Cohen nachweislich gelesen hat, sowie Widmungsexemplare.

21 Ders., Kleinere Schriften, I, 9 u. 25 (lat.) sowie 50 u. 72 (dt.). 
folgenden Schrift Die platonische Ideenlehre, psychologisch entwickelt von $1866^{22}$ rücken sie unmittelbar nebeneinander. „Es war meistens“, so wird Herder zitiert, „Ein neues Bild, Eine Analogie, Ein auffallendes Gleichnis, das die größten und kühnsten Theorien geboren." ${ }^{23}$ Und Cohen zieht den Schluss: „Ein plötzliches Bild ist der psychische Antrieb, die Wahrheit anschaulich zu denken, und im späteren Denken wird das ursprüngliche Bild geklärt und bewährt.“ ${ }^{24}$ Es ist der Ansatz zu einer Theorie der „Intuition“, die er dann - da er sie als Leistung einer „Synthese“ auffasst - einer komplexen „psychologischen Analyse“ unterziehen kann. ${ }^{25}$ Seine Absicht ist, die „Entdeckung“ der Ideenlehre als einen „,psychischen Proze $3^{\text {“ in }}$ der Person Platons nachzuzeichnen. ${ }^{26}$ Etymologische Überlegungen führen ihn zur Parallelsetzung des griechischen idea mit dem lateinischen visus, dem deutschen Gesicht und dem hebräischen chason für prophetische Einsicht. Die „Schau“ des Philosophen und Propheten ist es, wodurch „Entdeckungen“ gemacht werden, so auch die der Ideenlehre.

Diese sprachübergreifende Parallele zwischen Ideenschau und Prophetie hat Cohen lebenslang beschäftigt. Sie stand ihm früh in voller Klarheit vor Augen. Die Passage seiner Dissertation, wo er sie erstmals ausspricht, hat er (auf Deutsch) nahezu wörtlich in Die platonische Ideenlehre übernommen, nun in direktem Bezug zu dem Herderzitat und im Zentrum seiner Deutung der platonischen Idee. ${ }^{27} \mathrm{Neu}$ ist, dass Cohen das Wort „Idee“ und seine anderssprachigen Analoga nach ihrem metaphorischen Gehalt auszulegen beginnt, obwohl er den Begriff „Metapher“ nicht verwendet. „Idea“, ,visus“, „Gesicht“" und „,chason“ sind Vollzugsmetaphern dessen, was oben „Geist" hieß. Idee ist demnach wesentlich „Methode“, eine „lebendige [...] Grund- und Wesens-Anschauung"; durch sie ist Platon - und das bleibt ein Fixum in Cohens Werk - der „frühe Ahnherr der intellectuellen Anschauung, des transscendentalen Idealismus“. ${ }^{28}$ „Ideen“ im Plural realisieren „Entdeckungen“; ihr Zentrum aber haben sie in der singulären „Idee“, dem reflexiven Entdecken des Entdeckens selbst. Jedes Entdecken ist ein Sich-Aussprechen: eine psychophysiologische Performanz, zu der das leibliche Bilden von Wortlauten ebenso gehört wie die klanglose Bewegung einer ,inneren“ Vorstellungsmechanik.

Auch zu dieser Konsequenz gelangt Cohen erst über mehrere Stufen. In Platons Ideenlehre betont er zwar zweimal, wie lebendig in Platon die ,innere Sprachform“ sei, und bringt Beispiele, wo dieser „die Worte [...] wie flüssiges Metall faßt, das sich in die elastischen Windungen seiner Gedanken schmiegsam fügen muß“، ${ }^{29}$ Die dahinter stehende Sprachpsychologie wird jedoch erst in Heinrich Heine und das Judentum (1867), Mythologische Vorstellungen von Gott und Seele (1868/69) und vor allem in Die dichterische Phantasie und der Mechanismus des Bewußtseins (1869) entwickelt. Sein Sprachmaterial hatte Cohen bisher aus der klassischen Philologie und ergänzt es nun durch die neuere Indogermanistik, Steinthals Analyse hebräischer Quellen und durch die Mythologieforschungen Jacob Grimms, Adalbert

22 Ders., Die platonische Ideenlehre, psychologisch entwickelt, in: ders., Kleinere Schriften, I, 101191.

23 Ders., Kleinere Schriften, I, 140, zit. aus: J. G. Herder, Vom Erkennen und Empfinden der menschlichen Seele. Bemerkungen und Träume, Riga 1778, 4 f.

24

Ebd., 141.

25 Ebd., 106 u. 108

26 Ebd., 104 u. 106.

27 H. Cohen, Kleinere Schriften, I: „De antinomia“, 25 (lat.) u. 72 (dt.); ders., Die platonische Ideenlehre, a. a. O., 139.

28 Ders., Die platonische Ideenlehre, a. a. O., 144, 158, 137.

29 Ebd., 146; vgl. 136, 147, 152, 156. 
Kuhns und Wilhelm Mannhardts. ${ }^{30}$ Sein zweites, auf Dauer noch wichtigeres Standbein sind die physiologisch-neurologischen Theorien von Johannes Müller, Rudolph Hermann Lotze und Hermann von Helmholtz ${ }^{31}$, dann - etwas mehr im Hintergrund - die Halluzinationslehre des Psychiaters Wilhelm Griesinger sowie die biologische Zelltheorie von Matthias Jacob Schleiden und Theodor Schwann. ${ }^{32}$

Der zentrale Begriff dieser Sprachwissenschaft ist die „Anschauung der Anschauung“, eine Weiterentwicklung von Herbarts Begriff der "Apperzeption“. ${ }^{33}$ Cohens nennt als seine Anknüpfungspunkte vor allem das Kapitel „Geist und Sprache“ in der ersten Auflage von Moritz Lazarus' Leben der Seele (1856/57), und Chajim Steinthals Buch Grammatik, Logik und Psychologie (1855). ${ }^{34}$ Sprache ist, mit Steinthals Paraphrase der berühmten Definition Humboldts gesagt, ,wesentlich nur Sprechen; und soll von ihrem Wesen und Ursprung gehandelt werden, so darf sie nicht als gegebnes Mittel, sondern muss als geistige Tätigkeit genommen werden“. ${ }^{35}$ Sie ist ein immer neues „Hervortreten“ ${ }^{63}$ Ein physiologischer Reflex macht den Anfang: Eindrückliche „Anschauungen“, so Steinthal, veranlassen das Ausstoßen eines reaktiven Lauts. Das wird sich bei erneuter Anschauung wiederholen, und so assoziieren sich Anschauung und Laut. Schließlich weckt umgekehrt das Hören des Lautes die ehemalige Anschauung. Beim Menschen wandelt sich diese Konditionierung zur Fähigkeit der „Erinnerung“. Zur Darstellung dieser Wandlung dient der „im Geiste der Hegelschen Philosophie“ entwickelte Begriff einer „Anschauung der Anschauung““. ${ }^{37}$ Der Anlass liegt zwar wieder in der Anschauung eines Äußeren. Aber sie aktiviert diesmal nicht nur eine gespeicherte Struktur von Reflex- und Assoziationsbewegungen. „Anschauung der Anschauung“ bedeutet ein abstandhaltendes Betrachten der konditionierten Anschauung. Die „Seele“, so nennt Steinthal den Akteur des Betrachtens, ,sieht also im Aeussern nicht mehr bloss das Aeussere, sondern zugleich ihr Inneres; [...] d. h. sie wird sich ihrer selbst bewusst“. Das Vergangene wird ,wie ein verborgener Schatz aus der Tiefe der Seele an das Licht" gehoben. ${ }^{38}$ Das schafft die Möglichkeit für einen „Uebergang der Seele in den Geist“. ${ }^{39}$

30 Vgl. H. Cohen, Mythologische Vorstellungen von Gott und Seele; ders., Die dichterische Phantasie; beide in: ders., Kleinere Schriften, I, passim. Zu ästhetischen Referenzautoren (vor allem Friedrich Theodor Vischer) vgl. E. Gamba, La legalità del sentimento puro, Mailand 2008, 19-51.

31 Vgl. H. Cohen, Mythologische Vorstellungen, a. a. O., bes. 303-307 mit dem Kommentar des Bearbeiters; sowie ders., Die dichterische Phantasie, a. a. O., bes. 412-419.

32 Ders., Die dichterische Phantasie, a. a. O., 390-392 sowie 449 mit Kommentar.

33 Vgl. ebd., 426 ff., Quellenhinweise im Kommentar: 427; vgl. zum Folgenden H. Wiedebach u. A. Winkelmann (Hg.), Chajim H. Steinthal: Sprachwissenschaftler und Philosoph im 19. Jahrhundert, Leiden 2002, bes. 100-104.

34 Vgl. M. Lazarus, Das Leben der Seele in Monographien über seine Erscheinungen und Gesetze, Berlin 1856/57, Bd. 2, 3-258; vgl. H. Cohen, Die dichterischer Phantasie, a. a. O., 386 ff.; H. Steinthal, Grammatik, Logik und Psychologie, ihre Prinzipien und ihr Verhältnis zueinander, Berlin 1855.

35 H. Steinthal, Der Ursprung der Sprache im Zusammenhang mit den letzten Fragen alles Wissens, 4. Aufl., Berlin 1888, 59 f.

36 Ders., Grammatik, Logik, a. a. O., 295.

37 Ebd., 295 ff.; der Hinweis auf Hegel in: H. Steinthal, Der Ursprung der Sprache, a. a. O., 121; vgl. G. W. F. Hegel, Enzyklopädie der philosophischen Wissenschaften im Grundrisse [1830], §§ 452 ff. über „Erinnerung“ und „Einbildungskraft“; dazu M. Ringmacher, Organismus der Sprache. H. Steinthals Weg von Humboldt zu Humboldt, Paderborn 1996, 104 f.

38 H. Steinthal, Grammatik, Logik, a. a. O., 297; vgl. Hegels Rede von einem „Schacht“ des „Ich“ oder der „Intelligenz“ in: ders., Enzyklopädie [1830], §§ 403, 453 f. u. ö.

39 H. Steinthal, Grammatik, Logik, a. a. O., 300. 
Diesen Übergang vollzieht die Sprache. Sie bringt die „Gesammtmasse“, die sich der Innensicht darbietet, zur Einheit. Die Masse bündelt sich an einem repräsentativen Zeichen und tritt der „seelischen“ Bewegtheit gegenüber. Beides, sowohl die Bündelung als auch das Heraustreten, leistet nach Steinthal erneut ein physiologischer Reflex, nämlich der „lautmalerische“, onomatopoetische Sprachlaut. ${ }^{40}$ Das Schauen der Seele wird hier seinerseits zum Auslöser. Dieser, mit Herder gesprochen, , besonnene ${ }^{641}$ Reflex ist das Urphänomen der Sprache. Er malt aus der „Gesammtmasse“ ein einzelnes eindrückliches Motiv in akustischer Gestalt. Der Laut „Wolf” malt nur die Anschauung „der Reissende“. ${ }^{42}$ Aber ihm assoziieren sich weitere Motive und formen die Vorstellung eines Tieres mit bestimmten Eigenschaften. So nimmt die „selbstthätige Entwicklung des Denkens“ ihren Anfang..$^{43}$ Der Laut „Wolf“ ist nun ein „Wort“; ,,der Reissende“" aber bleibt darin die primäre „,innere Sprachform““.44

Ein ungelöstes Problem ist die Seele, das neben dem physiologischen Reflexapparat zweite hypothetische Substrat der Sprachbewegung. Hinter ihr stehen Herbarts Metaphysik der Seelensubstanz und seine Theorie der Vorstellungen als „Selbsterhaltungen der Seele““. 45 Zwar nimmt Steinthal Kritik durchaus zur Kenntnis, das belegt seine Anzeige (1871) von Jürgen Bona Meyers Buch Kant's Psychologie. ${ }^{46}$ Aber er schätzt die zeitgenössischen Prinzipiendenker nicht sonderlich: Weder die „Materialisten“ noch „,die meisten Idealisten“ hätten ,gezeigt, daß sie eine Ahnung von dem Mechanismus des Bewußtseins [...] haben, und daß sie wüßten, wie die Analyse einer verwickelten psychologischen Thatsache anzugreifen sei “ ${ }^{47}$ - Cohen dagegen, in einer Besprechung desselben Buches im übernächsten Heft der Zeitschrift, erörtert genau die Prinzipienfrage. ${ }^{48}$ Aus seiner inzwischen ausgearbeiteten kantischen Perspektive kehrt er nicht nur (mit Meyer) von Herbarts Seelenmetaphysik zu Kant zurück, sondern unterstellt sogar Meyer noch den Fehler, ,hypostasirte Kräfte“ in der Psychologie anzunehmen statt rein funktionaler Hypothesen. ${ }^{49}$

Cohen setzt Steinthal und Lazarus seine Lesart des „quid juris?“ entgegen: Was erzeugt die Geltung von Aussagen dort, wo sie die Gestalt von theoretischen Urteilen annehmen, das heißt

40 Vgl. ebd., 309 f.; dazu und zum Folgenden vgl. M. Ringmacher, Organismus der Sprache, a. a. O., 99-126.

$41 \mathrm{Zu}$ Herders Bestimmung des Menschen durch „Besonnenheit“" vgl. ders., Abhandlung über den Ursprung der Sprache [1772], 2. Abschnitt, Stuttgart 1966, 24-43, bes. 28 ff.

H. Steinthal, Grammatik, Logik, a. a. O., 320; zur Etymologie vgl. 313 f.

43 Vgl. ebd., 323.

44 Ebd., 325.

45 Vgl. J. F. Herbart, Allgemeine Metaphysik, nebst den Anfängen der philosophischen Naturlehre, $\S 220$, in: ders., Sämtliche Werke, hg. v. K. Kehrbach, Langensalza 1887-1912, Bd. 8, 83; ders., Selbsterhaltungen der Seele, in: ders., Psychologie als Wissenschaft, neu gegründet auf Erfahrung, Metaphysik, und Mathematik, § 153, in: ders., Sämtliche Werke, a. a. O., Bd. 6, 453.

H. Steinthal, [Rez.] Jürgen Bona Meyer, Kant's Psychologie (Berlin, Wilhelm Hertz 1870), in: Zeitschrift für Völkerpsychologie und Sprachwissenschaft, 7 (1871), H. 1, 110 f.

Ebd., 111.

H. Cohen, [Rez.] Jürgen Bona Meyer, Kant's Psychologie, in: Zeitschrift für Völkerpsychologie und Sprachwissenschaft, 7 (1871), H. 3, 320-330. Der sehr aufschlussreiche Text ist in den bisherigen Sammlungen nicht enthalten und wird erst wieder abgedruckt in: H. Cohen, Kleinere Schriften, II (Werke, 13).

49 Ebd., 326. - Das Heft 3/1871 der Zeitschrift für Völkerpsychologie und Sprachwissenschaft enthält (249-296) auch die für Cohens Kantianismus wichtige Stellungnahme: H. Cohen, Zur Controverse zwischen Trendelenburg und Kuno Fischer, demnächst in: ders., Kleinere Schriften, II (Werke 13). Im Jahr 1871 erschien außerdem die erste Auflage von: Kants Theorie der Erfahrung, Werke, 1.3. 
in der Wissenschaft? Unter dieser Frage wirken seine Lehrer naiv. Sie übertragen, überspitzt gesagt, den Modus mythischen Erzählens von ihren literarischen Quellen auf die Hermeneutik von deren Entstehung. Sie schreiben ein zwischen Leib und Seele spielendes Ursprungs- und Überlieferungsdrama der Sprache statt Wissenschaft. Zwar hat auch Cohen seine Quellen literarisch definiert. „Nicht die Sterne am Himmel sind die Objecte“, die es zu betrachten gilt, sondern ,die obersten Grundsätze einer in gedruckten Büchern wirklich gewordenen Erfahrung“", so heißt es $1877 .{ }^{50}$ Aber er beschränkt sich zunächst auf die Literatur, die das $\lambda$ órov dióoval, die logische Rechtfertigung zu ihrer Aufgabe macht. Philosophie wird Grundlegung der Wissenschaft. Sie nimmt, als Rechtfertigungsreflexion, die sich zugleich selbst rechtfertigt, die Kontur ihres Zielbegriffs an und wird ebenfalls Wissenschaft. Wo sie sich selbst konsolidiert, kennt sie keine Sprachseele: Ihre Grundlegungsakte sind Ausdruck logischer und nicht sprachlicher Sachverhalte. ${ }^{51}$ Ihr Agens ist auf diesem Teil des Weges das „transzendentale Subjekt“ oder - in Cohens Logik der reinen Erkenntnis von 1902 - das „reine Denken“.

Nicht dass Cohen die Psychologie irgendwann verabschiedet hätte. Schon die Besprechung von Meyers Buch lässt ahnen, dass die Frage nach der „Einheit des Bewußtseins“ auf der Tagesordnung bleibt. ${ }^{52}$ Ganz deutlich wird ihm selbst das aber erst sechs Jahre später. Er hält im Wintersemester 1877/78 erstmals eine dreistündige Psychologievorlesung. Hier gelangt er zu der von nun an gültigen Verknüpfung: „Den Schwerpunkt für die Psychologie als philosophische Disciplin lege ich in die Direction des Begriffs der Einheit des Bewußtseins; und diesen Zusammenhang denke ich deutlich und bestimmt herauszubringen. ${ }^{653}$ Dem sollte sich zuletzt auch der nie geschriebene Abschluss seines Systems der Philosophie widmen: eine, wie Fritzsche mitteilt, Psychologie des reinen Bewußtseins. ${ }^{54}$ Bis zum Schluss hielt Cohen daran fest, diese ,hodegetische Enzyklopädie des Systems“ noch auszuarbeiten. ${ }^{55}$ - Zur Psychologie aber gehört die Physiologie. In ihr hat jede systematische Psychologie „ihre untere Grenze“. Wo „Elemente der Physiologie“ ins Spiel kommen, „muß sich die psychologische Charakteristik von der Physiologie abhängig machen“. ${ }^{56}$ Die Menge an Fachliteratur, die Cohen zur Kenntnis nimmt, ist beachtlich. Allein die seit 1890 erscheinende Zeitschrift für Psychologie und Physiologie der Sinnesorgane von Hermann Ebbinghaus und Arthur König hat er 10 Jahre lang privat erworben und durchgearbeitet. ${ }^{57}$ Und es ist kein Bekenntnis aus momentaner Gestimmtheit, dass er 40 Jahre nach seinem Abrücken von der Leib-Seele-Theorie seiner sprachwissenschaftlichen Lehrer eine Abhandlung über altgrie-

50 H. Cohen, Kants Begründung der Ethik [1877], 20 u. 27.

51 Ders, Logik der reinen Erkenntnis, Werke, 6, 47 f.; vgl. H. Holzhey, Cohen und Natorp, Basel/Stuttgart 1986, Bd. 1, 98.

52 H. Cohen, [Rez.] Meyer, a. a. O., 321.

53 Brief Cohens an August Stadler vom 8.11.1877, Hermann Cohen-Archiv Zürich, Veröffentlichung im Rahmen der Werkausgabe geplant.

54 R. A. Fritzsche, Das Judentum im deutschen Denken. Hermann Cohen, in: Der Jude, 7 (1923), 429-439, hier: 438.

55 Zit. in: H. Cohen, Ästhetik des reinen Gefühls, Bd. 2, Werke, 9, 432; vgl. ders., Logik der reinen Erkenntnis, a. a. O., 609-612; zum Arbeitsplan den Brief an Natorp vom 10.6.1917, abgedruckt in: H. Holzhey, Cohen und Natorp, a. a. O., Bd. 2, 480.

56 H. Cohen, Ethik des reinen Willens, a. a. O., Vorrede zur 2. Aufl., 1907, XII f.; vgl. zum ethischen Kontext H. Wiedebach, Physiology of the Pure Will: Concepts of Moral Energy, in: Hermann Cohen's Ethics, hg. v. R. Gibbs, Leiden 2006, 85-103.

57 Vgl. dazu: Die Hermann-Cohen-Bibliothek, Werke, Suppl. 2, 1fd. Nr. 877-894; weitere Verweise im Register. 
chische Leiblichkeitsauffassungen ausführlich würdigt und dabei feststellt, der „Schwerpunkt aller philosophischen Interessen“ (!) liege „noch heute, wie damals, [...] in den Fragen des Bewußtseins im Verhältnis zur biologischen Materie“".58

\section{Der logische Anschlusspunkt Gottes}

Am 22. Januar 1876, Lessings Geburtstag, vollzog Wilhelm I. von Preußen Cohens Berufung zum Ordinarius in Marburg als Nachfolger von Friedrich Albert Lange und machte ihn damit zum ersten jüdischen Philosophieprofessor in Deutschland. ${ }^{59}$ Die bedeutendste Leistung seiner fast 40 Marburger Jahre ist das System der Philosophie, von dessen vier geplanten Teilen drei erschienen: Logik der reinen Erkenntnis (1902, 1914²), Ethik des reinen Willens (1904, $1907^{2}$ ) und Ästhetik des reinen Gefühls (1912). ${ }^{60}$ Jeder begründet eine „selbständige“ Richtung des Bewusstseins in Anbindung an ein kulturelles „Faktum“: denkende Erkenntnis in Anbindung an mathematische Naturwissenschaft, sittliches Handeln in Anbindung an reine Rechtswissenschaft, ästhetisches Gefühl in Anbindung an Kunst. In all diesen Jahren brach Cohens Engagement für das Judentum nie ab, aber es lief seit dem Austritt aus dem Rabbinerseminar gleichsam neben der systemphilosophischen Arbeit her. In seinen letzten 15 Jahren führte er die beiden Bemühungen zusammen.

Das konnte er abschließend erst leisten, als ihm die Ästhetik des reinen Gefühls, an der er seit etwa 1908 arbeitete ${ }^{61}$, dem Grundgedanken nach vor Augen stand. Denn das entscheidende Brückenglied zwischen System und Glaube ist Cohens Poetologie, eine kritische Wiederaufnahme von Begriffen aus Die dichterische Phantasie und der Mechanismus des Bewußtseins von 1869. Am wichtigsten werden zum einen die Dramaturgie als Basis für den religiösen Mitleidsbegriff, zum andern und vor allem die Theorie der Lyrik als einem bekennenden Sprechen im Modus der Sehnsucht. ${ }^{62}$ Die ersten Dokumente sind die zweite Fassung von Religiöse Postulate von 1909 und Innere Beziehungen der Kantischen Philosophie zum Judentum sowie Die Einheit des Herzens bei Bachja von $1910 .{ }^{63}$ Die Mitleidstheorie nimmt erst nach Cohens Russlandreise vom Mai 1914, das heißt mit seinem Buch Der Begriff der Religion im System der Philosophie von 1915, Gestalt an. ${ }^{64}$ Die Durchführung für die Lyrik

58 H. Cohen, Über die Bedeutung einer philosophischen Jugendschrift Ludwig Philippsons [1911]. - Der physiologische Hauptteil von Cohens Text wurde in den Jüdischen Schriften weggelassen, jetzt wieder veröffentlicht in: H. Cohen, Kleinere Schriften, IV, Werke, 15, 565-604, zit. 599. - Die

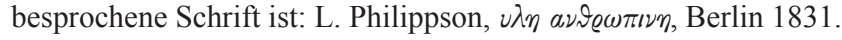

59 Briefe Cohens an August Stadler vom 5.2.1876, Hermann Cohen-Archiv (Zürich), und an Leopold Zunz vom 6.2., The National Library of Israel (Jerusalem), Zunz-Archiv: Arc. 4 792, G 10 (Sammlung Wiedebach, Göppingen: +U/8).

60 Wann genau er die Absicht zu einem eigenen System fasste, ist unklar. Noch 1902 verhielt er sich zögernd; vgl. H. Holzhey, Cohen und Natorp, a. a. O., Bd. 2, 277; G. Edel, Der Zusammenhang der Systemteile in ihrer methodischen Verknüpfung, in: Hermann Cohen und die Erkenntnistheorie, hg. v. W. Marx u. E. W. Orth, Würzburg 2001, 110-122, hier: 111.

61 Zur Entstehungsgeschichte der Ästhetik des reinen Gefühls vgl. demnächst die „Einleitung“ von H. Wiedebach zur Neuauflage von: H. Cohen, Werke, 8.

62 Vgl. H. Wiedebach, Aesthetics in Religion: Remarks on Hermann Cohen's Theory of Jewish Existence, in: The Journal of Jewish Thought and Philosophy, 11 (2002), 63-73.

H. Cohen, Kleinere Schriften, IV, Werke, 15, 155-160, 309-345, 415-428.

64 Ders., Werke, 10, bes. 76-80 u. ö.; zuletzt vor allem in: ders., Religion der Vernunft, a. a. O., bes. 19 ff. u. $160 \mathrm{ff}$. 
findet man in dem Aufsatz Die Lyrik der Psalmen von $1914^{65}$ und dann vor allem in Cohens Theorie der Versöhnungsliturgie, dem Zentrum seiner Religion der Vernunft aus den Quellen des Judentums (posthum 1919). ${ }^{66}$

Natürlich verband Cohen bereits früher Motive der jüdischen Tradition mit systemphilosophischen Erörterungen. Es ließe sich aber, so meine ich, nachweisen, dass das auf wechselseitiger Entleihung, nicht auf einer Verknüpfung in systematischer Einheit beruhte. Für Cohen ist Judentum eine Religion. Daher liegt das entscheidende Problem im Gottes- und, damit zusammenhängend, im Offenbarungsbegriff: „In dem Verlangen nach Gott besteht die Religion. In dem Verlangen nach einem Wesen außer dem Menschen, aber für den Menschen besteht sie. “67 Um dem philosophisch nachzudenken, genügt es nicht, Gott in der Mitte des Systems einzuführen, auch wenn dort der Mensch thematisch wird. Solange nicht von der methodischen Grundlegung, das heißt von der Erkenntnislogik her, eine Homologie zwischen Religion und System entdeckt wird, bleibt das Verständnis von Cohens Religionsphilosophie, wie Werner Flach treffend bemerkt hat, auf biographische „Motivationsforschung“ angewiesen. ${ }^{68}$

Die Gottesidee in der Ethik des reinen Willens (1904) zeigt das gut. Gott hat in der Logik der reinen Erkenntnis (1902) keine Grundlegungsbedeutung. In der Ethik dagegen ist er eine geschichtsphilosophische Idee, die dem „Ideal“ - einem Leitbild der „Ewigkeit“ für die Gestaltung ethischer Wirklichkeit - die analoge Erhaltung der Natur verbürgt: „Gott bedeutet, daß die Natur Bestand hat, so gewiß die Sittlichkeit ewig ist. " ${ }^{69}$ Man mag das systematisch für angreifbar halten. ${ }^{70}$ Vorliegend kommt es darauf an, dass Cohen selbst diesen Gott in der Religion der Vernunft aus den Quellen des Judentums als eine Entlehnung bezeichnet hat: „Wie die wissenschaftliche Ethik alle die literarischen Quellen treulich benutzen muß, so haben wir aus dem Monotheismus diesen Gott in unsere Ethik verpflanzt. Und dennoch ist dieser aus der Religion entlehnte Gott doch nur kraft der Berührung, welche zwischen dem Monotheismus und der Sittenlehre sich vollzieht, ein ethischer Gott, noch nicht aber der eigentliche Gott der Religion." "71 Um diesen eigentlichen Gott erkennen zu können, muss das System der Philosophie unter dem Licht jenes ,Verlangens nach Gott“ noch einmal, und zwar als Ganzes, durchlaufen werden. Das ist Inhalt der Religion der Vernunft von 1919. Die logische Eintrittsschwelle jedoch überschritt Cohen in der Arbeit an dem großen Aufsatz Charakteristik der Ethik Maimunis von 1908.

65 Ders., Kleinere Schriften, V, Werke, 16, 163-198. Der Text blieb zu Cohens Lebzeiten unveröffentlicht. Seine Datierung auf 1914 folgt der Vermutung von Bruno Strauß, der als Letzter den inzwischen verlorenen Nachlass Cohens benutzt hat (vgl. Werke, 16, 164). Dieter Adelmann hat eine Umdatierung in die Nähe des Aufsatzes Der Stil der Propheten von 1901 vorgeschlagen (vgl. D. Adelmann, „Reinige dein Denken“: Über den jüdischen Hintergrund der Philosophie von Hermann Cohen, hg. v. G. Hasselhoff, Würzburg 2010, 125 f., 149 f.). Das ist nicht haltbar, eben weil Die Lyrik der Psalmen die in der Ästhetik des reinen Gefühls (1912) entwickelte Theorie der Lyrik voraussetzt, von der der frühere Text über die Propheten unberührt ist.

66 H. Cohen, Religion der Vernunft, a. a. O., 247 f., zum (Sünden-),,Bekenntnis“ Kap. XII: „Der Versöhnungstag“, 252-275.

67 Ders., Der Begriff der Religion im System der Philosophie [1915], Werke, 10, 138.

68 W. Flach, Hermann Cohens Grundlegungskonzept und sein monotheistischer Gottesbegriff, in: Man and God in Hermann Cohen's Philosophy, hg. v. G. Gigliotti, J. Kajon, A. Poma, Padua 2003, 41-50, hier: 42; zur Abwehr der Biographik vgl. 46 f.

69 H. Cohen, Ethik des reinen Willens, a. a. O., 446.

70 Vgl. H. Holzhey, Gott: die Zukunft einer Illusion. Religionskritik bei Freud und Cohen, in: Man and God in Hermann Cohen's Philosophy, a. a. O., 51-62, bes. 56-60.

71 H. Cohen, Religion der Vernunft, a. a. O., 25. 
Er schrieb an dem Text seit Sommer $1905 .{ }^{72}$ Eine erste Fassung konnte nicht termingerecht erscheinen, und Cohen nahm 1906 eine Änderung vor: „Ich möchte den Maimonides durch den Zusatz ergänzen, daß seine negativen Attribute Gottes, und nur diese sind ihm ja zuläßig, viel mehr limitierende Ursprungsprädikate sind. Eine sehr angenehme Beschäftigung. ${ }^{\text {“73 }}$ Er kam, so heißt es im Aufsatz selbst, ,,an einen Punkt, an dem ich einem Grundgedanken meiner eigenen systematischen Logik nachgehe“. Ein solches „selbständige[s] Interesse der Spekulation“ gehört, so erklärt er, zu ,alle[r] Geschichte der Philosophie“; sie „sollte“ geradezu damit „,behaftet sein““ ${ }^{74}$ Cohens „,selbständiges Interesse“ ist die Deutung Gottes mithilfe seiner „Logik des Ursprungs“ aus der Logik der reinen Erkenntnis. ${ }^{75}$ Im vorliegenden Zusammenhang ist vor allem die Konsequenz wichtig. Durch die Assimilierung der Erkenntnislogik an einen religiösen Bekenntnisinhalt - für Maimonides ist der Glaube an die Schöpfung ausdrücklich nicht philosophisch begründbar ${ }^{76}$ - bereitet Cohen den Boden für seine eigene Metamorphose des Erkenntnisbegriffs, nämlich für die Ineinssetzung von Erkennen und Bekennen, genauer: von Erkenntnis und Liebe. ${ }^{77}$

Dieser veränderte Erkenntnisbegriff begründet Cohens späte Religionsphilosophie, die sich in der Folge den Gesamtstoff des philosophischen Systems in einer Art zweitem Durchgang zueignen wird. Den Schritt zur Ethik hält er mit der Verbindung von Erkenntnis und Liebe schon für getan. Er meint, das Gotteserkennen, welches Maimonides im Sinn einer aristotelischen theoria entwirft, als eine zuletzt ethische „Selbstannäherung“ (hebr. hitkarbut) ${ }^{78}$ des Menschen an Gott in Wollen und Tun auslegen zu dürfen. Das verleiht diesem Erkennen eine Eigenschaft, die Cohen im System vermieden hatte, nämlich die theoretische Erkenntnislogik ethisch zu normieren ${ }^{79}$ beziehungsweise im Gefühl zu verankern. In der Religionsphilosophie wird das zur Selbstverständlichkeit. Den Schritt zur Ästhetik geht Cohen in der Charakteristik allerdings noch nicht.

Einen Tag nach deren Absendung an den Drucker macht er sich auf Reisen, ,,aufgeregt, aber doch innigst bewegt von der Ansicht, die sich immer mehr mir bestärkte, daß sowohl die philosophische wie die jüdische Geistesart dieses unsres einzigen Mannes mehr als bisher nunmehr herausgestellt worden ist" ${ }^{\circ}{ }^{80}$ Man versteht die Erregung: Zwei für Cohen existenzielle Bereiche der Auseinandersetzung sind in der Auslegung eines mittelalterlichen Denkers zusammengekommen.

72 Vgl. die „Einleitung“ zu: Kleinere Schriften, IV, Werke, 15, XXIII-XXVII.

73 Brief an Ernst Cassirer vom 22.7.1906. Es dürfte sich vor allem um Teil IV der 1908 publizierten Charakteristik handeln (vgl. H. Cohen, Kleinere Schriften, IV, Werke, 15, 206-223, bes. den Bezug auf die Logik, 219; zu Cohens Briefen an Cassirer vgl. die CD als Beigabe zu: E. Cassirer, Ausgewählter wissenschaftlicher Briefwechsel, hg. v. J. M. Krois, Hamburg 2009, Nachgelassene Manuskripte und Texte, 18). Eine Publikation im Rahmen der Cohen-Ausgabe ist geplant.

H. Cohen, Charakteristik, a. a. O., 219 u. Anm. 2 des Bearbeiters.

Vgl. M. Kavka, Jewish Messianism and the History of Philosophy, Cambridge 2004, 66-128; jüngst auch mehrere Arbeiten (A. W. Hughes, R. Erlewine, J. A. Diamond, G. Y. Kohler; mit Hinweisen auf weitere Literatur) in: The Journal of Jewish Thought and Philosophy, 18 (2010).

76 Vgl. Maimonides, Führer der Unschlüssigen, Buch II, bes. Kap. 25.

77 H. Cohen, Charakteristik, a. a. O., $227 \mathrm{ff}$.

78 Ebd., 228-232, 256; H. Cohen, Religion der Vernunft, a. a. O., 190, 364.

79 Vgl. H. Holzhey, Cohen und Natorp, a. a. O., Bd. 1, 321 f. u. 335 f.

80 Brief an Leopold Lucas vom 14.8.1906, in: H. Cohen, Briefe, a. a. O., 78 f. (nicht von 1908, wie dort angegeben; vgl. ders., Kleinere Schriften, IV, XXV, Anm. 2). 


\section{Einheit des Herzens}

Im Religiösen bleibt die Ethik der Maßstab. Aber die Religion stellt ihr das Vertrauen zur Seite. „Was die Religion Vertrauen nennt, das nennt die Ethik Denken, Erkenntnis der Idee.“81 Dieser lakonische Brückenschlag in dem Aufsatz Gottvertrauen von 1916 zieht bereits eine Summe aus der Zeit nach der Charakteristik. Seit 1909, in der erwähnten zweiten Fassung von Religiöse Postulate (Erstfassung 1907), nimmt Cohen nach langer Zeit wieder Motive der Sprachpsychologie Chajim Steinthals in seine Expositionen auf. ${ }^{82}$ Das geschieht in einem der von nun an häufigen Plädoyers für die hebräische Gebetssprache. Gleich sein erstes Beispiel ist das Benennen der göttlichen Einzigkeit im wichtigsten Satz des täglichen Gottesdienstes, im Schema 'Jisra' el (Deut 6,4: „Höre Israel, der Herr unser Gott, der Herr ist einzig, hebr. 'echad"): „Man muß Echad verstehen, in seinem ganzen sprachlichen Klima verstehen, um die Einzigkeit Gottes so zu denken, wie sie gefühlt werden muß. Hier liegen die Quellen des Steinthalschen Geistes, aus denen er das jüdischen Denken und Fühlen einheitlich mit der Psychologie der Sprache tief und nachhaltig befruchtet hat. “83 Dieses ,die Einzigkeit Gottes so zu denken, wie sie gefühlt werden muß“" von 1909 ist das neue Programm.

Der Satz verweist auf die Ästhetik des reinen Gefühls, den dritten Teil des Systems. Hier kommt die Sprachpsychologie erneut zur Geltung, aber nicht absolut genommen wie in den frühen Arbeiten. Im wissenschaftlichen Erkennen von Natur und Moral hat das ,reine Gefühl“ sein kritisches Rückgrat, seine „Vorbedingungen“. ${ }^{84}$ Auf deren Basis begründet Cohen seine Auffassung von der paradigmatischen Bedeutung der Poesie: eine „Art von neuer Logik“, die ,,in die anderen Künste herübergenommen“ wird. ${ }^{85}$ Methodisches Prinzip ist die „Verinnerlichung". Begriffe aus Naturerkennen und Ethik werden diesmal nicht auf ihre objektivgegenständliche Geltung hin beurteilt. Die ästhetische „Bewußtseinsrichtung“ setzt bei den Gefühls-,,Annexen“ des Denkens sowie des Wollens an und synthetisiert sie zu selbständigen Gestaltungskräften. ${ }^{86}$ Stehen sich zwei Worte in dem sie begleitenden Gefühl nahe, so lassen sie sich in einen poetischen Ausdruck bringen, der das eine zur Erläuterung oder Hervorhebung des anderen verwendet. Das von Steinthal übernommene Beispiel ist Psalm 19,6: Der Sonnenball geht auf, ,wie ein Bräutigam hervorgeht aus seinem Brautgemache / Er freut sich, wie ein Held, zu durchlaufen die Bahn“. ${ }^{87}$ Sonnenaufgang, Bräutigam und Held führen mit sich das Gefühl der Freude, und der Psalm kann sie daher einander assimilieren. Verinnerlichung ist - wie schon 1869 in Die dichterische Phantasie - „Vergleichung“. ${ }^{88}$ Die

81 Ders., Gottvertrauen [1916], Kleinere Schriften, VI, Werke, 17, 349.

82 Die Veränderungen sind textkritisch dokumentiert in: H. Cohen, Kleinere Schriften, IV, Werke, 15, 133160 .

83 Ders., Religiöse Postulate [erw. Fassung 1909], Kleinere Schriften, IV, Werke, 15, 158. Die erste Fassung von 1907 enthält weder diesen Hinweis noch das Plädoyer für die hebräische Sprache.

84 Ders., Ästhetik des reinen Gefühls (im Folgenden ÄrG, I u. II; Werke, 8 u. 9), I, 217-225.

85 ÄrG, I, 367.

86 Vgl. ebd., 143 ff. u. vor allem 363-369. Ein Vorbote dessen ist die Affekttheorie in: H. Cohen, Ethik des reinen Willens, a. a. O.; vgl. U. Renz, Affektivität und Geschichtlichkeit. Hermann Cohens Rehabilitierung des Affekts, in: Affekte und Ethik. Spinozas Lehre im Kontext, hg. v. A. Engstler u. R. Schnepf, Hildesheim 2002, 297-319.

87 ÄrG, I, 370; vgl. auch H. Cohen, Die Lyrik der Psalmen, Kleinere Schriften, V, Werke, 16, 178; zu Steinthal Anm. 2 des Bearbeiters.

88 ÄrG, I, 369-382; vgl. H. Cohen, Die dichterische Phantasie, Kleinere Schriften, I, 405; zur Quelle dieses Begriffs bei Steinthal vgl. 406, Anm. 2. 
poetische Gefühlsgestalt ist eine gegenüber der objektivierenden Erkenntnissprache „Zweite innere Sprachform". 89

In der Religion bekommt die Vergleichung eine eigene Bedeutung. Religiöses Denken ist für Cohen ein Vernunftgeschehen aus Vertrauen. Das Philosophische daran tritt im Kontrast hervor. In Anlehnung an Ernst Troeltsch fragt er einmal: „Kann und darf die Religionsphilosophie Religionswissenschaft werden?" ${ }^{\prime 90}$ Die Antwort lautet ,nein'. Weder die theoretische Religionswissenschaft noch die Religionsgeschichte, die er aus der zeitgenössischen „Religionsgeschichtlichen Schule“ kannte und schätzte, noch die Religionssoziologie können die philosophische Begründungsarbeit ersetzen. ${ }^{91}$ Die Philosophie muss die Vernunft als Vertrauensphänomen beglaubigen. ${ }^{92}$ „Was die Religion Vertrauen nennt, das nennt die Ethik Denken, Erkenntnis der Idee“, war der Satz von 1916. Oben zeigte er seine Bedeutung von der Ethik her: Wo diese ihren Gott dachte, stand daraufhin das Fühlen des 'echad (einzig!). Jetzt wird der Satz vom religiösen Gefühl her gelesen: Wo das Gottvertrauen sein 'echad fühlt, soll nun erkennend gedacht werden. Diese Umkehrung in vollem Ernst vollziehen heißt, ausschließlich vom Gefühl her Vergleichungen erproben. Und dies geschieht, obwohl Gott einzig ist, das heißt in der starken, vom Zahlbegriff gelösten Lesart ${ }^{93}$ : unvergleichbar mit allem Gegenstand möglicher Erkenntnis.

Genau dieses Paradox sichert dem Gefühlsvergleich seine Bedeutung, denn, pointiert gesagt: Von Gott lässt sich - eben wegen seiner Un-Vergleichbarkeit - überhaupt nur in Vergleichungen, sprich in Metaphern sprechen, obwohl sie notwendig ,uneigentlich', ja geradezu ,missbräuchlich“ (katachrestisch) sind. „Die Einzigkeit Gottes so zu denken, wie sie gefühlt werden muß“, wird zur Methode der Glaubensphilosophie. Sie muss ihre Begriffe vom Gefühlsannex her finden, ein schwieriges Unterfangen, denn als Begriffe unterliegen sie dem Maßstab der Vernünftigkeit. Der Gefühlsstoff, an dem diese Art Denken ansetzt, kommt aus der religiösen Tradition, bei Cohen in einem hohen Maß aus dem täglichen Gebet. ${ }^{94}$ Das Ergebnis sind Begriffsworte der Vernunfterkenntnis, deren Gefühlsannexe dem Vertrauensgefühl gleichen, das die Worte der religiösen Tradition begleitet. Daher wird Cohens Religionsphilosophie zunehmend ein Kommentar traditioneller Quellen. Sie wagt, angeleitet durch die Theorie der Poesie, ein systematisches Reden von Gott in psychologischen, auch leiblichen Metaphern, ohne sie erkenntniskritisch als gegenstandshafte Wesenserkenntnisse gelten zu lassen. Dadurch aber wird nun der Mensch, der seine Religion fühlend denkt, zum eigentlichen Ziel der Einsicht. Theologische Metaphern sind Begriffsspiegel, in denen der, der sie gebraucht, seine

89 ÄrG, I, 382-387.

90 H. Cohen, Reflexionen und Notizen, Werke, Suppl. 1, Blatt 57 mit Kommentar in Anm. 1.

91 Cohen las religionsgeschichtliche und systematische Artikel (von Hermann Gunkel, Eduard Grafe, Martin Rade, Ernst Troeltsch u. a.) zum Beispiel in der ersten Auflage von Die Religion in Geschichte und Gegenwart (Tübingen 1909-1913; vgl. Die Hermann-Cohen-Bibliothek, Nr. 628 f.). Auch Rudolf Smends Lehrbuch der alttestamentlichen Religionsgeschichte (2. Aufl., Freiburg 1899) fand sein Interesse (Nr. 723). Wellhausens Arbeiten zur Geschichte Israels, Gfrörers Kritische Geschichte des Urchristenthums und anderes waren direkte Quellen für Cohens Theorie des Opfers und der Versöhnung (vgl. H. Cohen, Reflexionen und Notizen, Blätter 130 f., 148 f.; zur Soziologie vgl. seine Kritik an Troeltsch in: H. Cohen, Der ethische Monotheismus und seine soziologische Würdigung; ders., Der Prophetismus und die Soziologie [1917], Kleinere Schriften, VI, Werke, 17, 493-501 u. 503-510).

92 Vgl. H. Cohen, Der Begriff der Religion [1915], Werke, 10, 1-9; ders., Religion der Vernunft, a. a. O., 2-12.

93 Vgl. bereits ders., Logik der reinen Erkenntnis [1902, 1914²], Werke, 6, 170, 474.

94 Vgl. dazu vor allem ders., Reflexionen und Notizen, Einl., II-IV und zahlreiche Verweise im Register unter „Gebet“; bei den einzelnen Blättern die Nachweise zu Schriften Cohens. 
eigene Kontur erkennt. Gottes „Zorn“, seine „13 Eigenschaften“ der Güte ${ }^{95}$, ja seine Gesten werden Momente in einer Hermeneutik des vernünftigen Menschseins.

Ein eindrückliches Beispiel ist die Auslegung von Bachja Ibn Pakudas Buch der Herzenspflichten (2. Hälfte des 11. Jahrhunderts) in Cohens Aufsätzen Innere Beziehungen der Kantischen Philosophie zum Judentum und Die Einheit des Herzens bei Bachja von $1910 .^{96}$ Verglichen werden hier nichts Geringeres als der Vollzug der Einzigkeit Gottes und die Stiftung der Einheit im Herzen des Menschen. Eine Nähe zur Metaphorologie zeigt schon der Ausdruck „Menschenherz“, ein Leitbegriff für die ästhetische Liebe. ${ }^{97}$ Und sie bestätigt sich vollends durch die Konsequenzen, die Cohen diesem Lieblingsautor seiner Jugend entlockt. Wieder geht es um ein Gebet, nämlich die tägliche Bitte „Einige unsere Herzen, zu lieben und zu ehrfürchten deinen Namen“ unmittelbar vor dem „Höre Israel““.98 „Einigung des Herzens in der Einigung Gottes“: So lautet - in Cohens Übersetzung - Bachjas Formulierung, durch die er benennt, was beim Aussprechen der Bitte geschieht. ${ }^{99}$ Diesen Ausdruck wiederum kommentiert Cohen: „Die Einheit Gottes“ - und mit „Einheit“ meint er diesmal das hebräische jichud, welches das fast noch statisch anmutende 'echad zusätzlich dynamisiert - „bedeutet die Erkenntnis und das Bekenntnis seiner Einheit; in ihnen vollzieht sich für das religiöse Bewußtsein die Einigung und in ihr die Einheit Gottes selbst." ${ }^{100}$ Der Mensch also vollzieht die Einigung Gottes. Und genau ,in“ der Einigung Gottes - Cohen betont das „in“ ausdrücklich - geschieht die „Einigung des Menschenherzens“. ${ }^{101} \mathrm{Zu}$ einer Ver-Einigung kommt es indes nicht, denn - die Erkenntniskritik macht sich geltend - es bleibt die „Unterscheidung“ selbst „ewiger Dinge und Ideen [...] von der Grundidee des ewigen, des einzigen Gottes“ “. ${ }^{02}$ Das „einige Herz“ ist folglich der Begriff für eine intime Selbstannäherung im Bewusstsein des bleibenden Abstandes. So konstituiert sich statt Identität eine „Korrelation zwischen der Einheit des Herzens und der Einheit Gottes“. ${ }^{103}$ Damit führt ein religiöses Gefühl auf eigenem Weg zurück zum logischen Begriff der Einheit und vertieft ihn durch seine Eigenart.

PD Dr. Hartwig Wiedebach, Universität Zürich, Hermann Cohen-Archiv, Zürichbergstrasse 43, 8044 Zürich, Schweiz

95 So die traditionelle Zahl der so genannten „Attribute der Handlung“ in Auslegung von Ex 34,6-7 (Babyl. Talmud, Tr. Rosch Ha-Schana 17b); vgl. vor allem H. Cohen, Religion der Vernunft, a. a. O., 109-115.

${ }^{96}$ H. Cohen, Kleinere Schriften, IV, bes. 322 f. u. 421-425.

97 Vgl. ÄrG, I, 224.

98 Nach Ps 86,11, hier zit. nach: H. Cohen, Der Nächste, Kleinere Schriften, V, Werke, 16, 75 (im Bachja-Aufsatz nicht wörtlich zitiert, vgl. ders., Kleinere Schriften, IV, 419 f.).

99 Ders., Die Einheit des Herzens, Kleinere Schriften, IV, 421. Cohen liest die hebräische Übertragung des arabischen Originals durch Jehuda Ibn Tibbon (in: Choboth ha-L'baboth, Lehrbuch der Herzenspflichten, hg. v. M. E. Stern, Wien 1853, hier: 356). Sterns deutsche Übersetzung zitiert er nicht.

100 Ebd., 422. - Zu hebr. jichud vgl. H. Cohen, Religion der Vernunft, a. a. O., 48. Wie wichtig dieses Wort für Cohens Bachja-Auslegung war, zeigt seine Polemik gegen den Arabisten und Talmudisten Wilhelm Bacher, der ihn durch Hinweise auf das arabische Original kritisierte. Die Einheit des Herzens bei Bachja ist insgesamt eine Verteidigungsschrift gegen Bacher (vgl. H. Cohen, Kleinere Schriften, IV, 417 f. und Einl., XXXI; zu Bachjas Begriffen vgl. F. Albertini, The influences of Saadya Gaon and Bachya Ibn Paquda on the Cohenian Concept of God's Uniqueness, in: Man and God in Hermann Cohen's Philosophy, a. a. O., 183-198, bes. 194 ff.).

101 H. Cohen, Die Einheit des Herzens, a. a. O., 422.

102 Ebd., 427.

103 Ebd. 


\begin{abstract}
Hermann Cohen's passionate philosophizing begins with a departure from the letter of the rabbinical doctrine of revelation. Initially his Science of Reason is shaped by a psychology of language based on Plato, Herder, Humboldt, and Steinthal. Later the influence of Kant is prevalent. In the end Cohen's System of Philosophy becomes the foundation upon which he reappropriates the sources of Judaism in their literalness. His program from 1908/09 onwards is to "think the uniqueness of God as it must be felt." A distinctive metaphorics comes into being: a hermeneutics of being human anchored in Reason through comparison with an incomparable God. The critical edition of his works illuminates this philosophical process by means of details previously not taken into account.
\end{abstract}

